\title{
Design of Strained Ge Schottky Diode on Si Substrate for Microwave Rectifier Circuit
}

\author{
Wei-Feng Liu, Xue-Mei Wu ${ }^{D}$, Jian-Jun Song ${ }^{(D}$, Xin-Yan Zhao, and Rong-Xi Xuan \\ Key Lab of Wide Band-Gap Semiconductor Materials and Devices, School of Microelectronics, Xidian University, \\ Xi'an 710071, China \\ Correspondence should be addressed to Xue-Mei Wu; 1293896044@qq.com
}

Received 9 October 2019; Revised 20 December 2019; Accepted 2 January 2020; Published 26 February 2020

Guest Editor: Renu Choudhary

Copyright ( 92020 Wei-Feng Liu et al. This is an open access article distributed under the Creative Commons Attribution License, which permits unrestricted use, distribution, and reproduction in any medium, provided the original work is properly cited.

In recent years, wireless energy transmission technology has developed rapidly and has received increasing attention in the industry. For microwave wireless energy transfer system applications, Ge Schottky diodes as the core components of the rectifier circuit are commonly used. Compared with Ge semiconductor, strained Ge semiconductor on Si substrate has the advantages of compatibility with Si process, low cost, and high electron mobility. It is an ideal replacement material for Ge semiconductor applications. In view of this, based on the model of the relationship between the performance of strained Ge semiconductor on $\mathrm{Si}$ substrate Schottky diodes and the geometric parameters of the device and the physical parameters of the material, Silvaco TCAD and ADS simulation software are jointly used to propose a novel strained Ge semiconductor on Si substrate Schottky diode for microwave rectification circuit. Simulation results show that the strained Ge semiconductor on Si substrate Schottky diode has a rectification efficiency of $70.1 \%$ when the input of the rectifier circuit is $20 \mathrm{dBm}$, the load resistance is $R=1000 \Omega$, and the load capacitance is $C=100 \mathrm{pF}$. Compared with traditional Ge Schottky diodes, this optimal operating point is closer to a low energy density, which is beneficial to a wide range of energy absorption. Studies have shown the feasibility of replacing Ge Schottky diodes. The research in this paper can provide valuable reference for the design and development of the core components of the rectifier circuit of the microwave infinite energy transmission system.

\section{Introduction}

Wireless energy transmission technology refers to transmission from the source to the receiver without the need for a conventional wire. Currently, the wireless energy transmission is divided into two categories according to the nearfar field. The near-field infinite energy transmission includes electromagnetic induction transmission and electromagnetic resonance transmission. The former uses a magnetic field as a medium and realizes wireless transmission through a transformer coupling, and the transmission power is large. The latter causes electromagnetic resonance when the natural frequency of the antenna is consistent with the frequency of the transmitting field, and strong electromagnetic coupling occurs to achieve wireless transmission, and the power is up to kilowatts. The far-field wireless energy transmission is mainly microwave wireless power transfer
(MWPT), which converts microwave energy into electrical energy and then transmits it to the destination through free space. It can be converted into DC power and supplied to the load through rectification, thereby achieving nonphysical connected energy transfer [1].

As a kind of wireless power transmission technology, MWPT has the advantages of small transmission attenuation in the atmosphere, long transmission distance, and large transmission power. It is an industry with great application prospects. The transmitting end and the receiving end make up the MWPT. As shown in Figure 1, the transmitting end converts the DC energy into microwave energy and transmits the energy through the transmitting antenna. The receiving end functions to receive the microwave energy through the rectifying antenna converted to DC energy to supply the load [2-5]. Microwave source generates microwave energy (power) into free space 


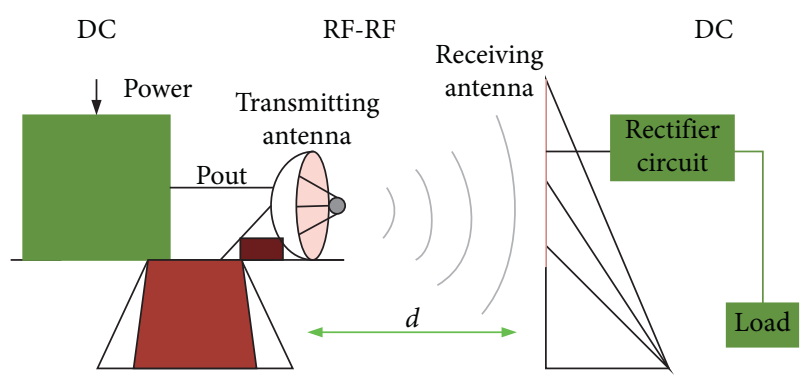

FIgURE 1: Schematic diagram of infinite energy transfer [1].

through transmitting antenna. The receiving antenna converts the captured energy into DC energy and delivers it to the rectifier circuit load.

At present, the core components used in the rectifier circuit of microwave wireless energy transmission system are commonly used by Agilent's HSMS-282X, HSMS285X, HSMS-281X, and HSMS-286X Ge-based Schottky diodes, which can cover low energy density to high energy density applications [6-8]. Compared with Ge semiconductor, strained $\mathrm{Ge}$ semiconductor on Si substrate (s-Ge/Si) has the advantages of compatibility with Si process, low cost, and high electron mobility [9-12]. It is an ideal replacement material for Ge semiconductor applications. As shown in Figure 2, Ge layer has tension. The thermal expansion coefficients of $\mathrm{Si}$ and $\mathrm{Ge}$ are different. The $0.2 \%$ tensile strain is introduced into the $\mathrm{Ge}$ epitaxial layer during annealing, which results in the change of the band structure and the enhancement of mobility.

In view of this, firstly, this paper uses $\mathrm{kp}$ perturbation theory and Monte Carlo simulation method to establish s-Ge/Si band structure and electron mobility model. On this basis, the Silvaco TCAD simulation tool is used, and a relationship model between s-Ge/Si Schottky diode performance and device geometry parameters and material physical parameters was established. An s-Ge/Si suitable for the MWPT system is the proposed Schottky diode device structure. The purpose of this paper is to explore the feasibility of s-Ge/Si replacement for Ge Schottky diodes and provide a valuable reference for the design and development of core components of MWPT system rectifier circuits.

\section{Strained Ge Semiconductor on Si Substrate Band and Electron Mobility}

Energy band structure and electron mobility are one of the theoretical foundations for research and design based on $\mathrm{s}-\mathrm{Ge} / \mathrm{Si}$ Schottky diodes. At present, the quantitative conclusions of s-Ge/Si semiconductor electron mobility are still lacking. Thus, this section first uses the kp perturbation theory to establish the s-Ge/Si band structure model (for details, see [11-14]) and further gives the electron mobility model of s-Ge/Si semiconductor. Under the one-

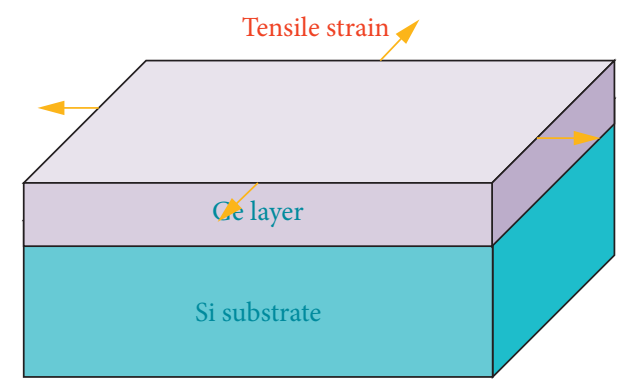

Figure 2: Typical strained $\mathrm{Ge}$ semiconductor on $\mathrm{Si}$ substrate semiconductor ( $\mathrm{Si}$ and Ge have different thermal expansion coefficients, and $0.2 \%$ tensile strain is introduced into the Ge epitaxial layer during annealing, resulting in band structure change and mobility enhancement).

electron approximation of periodic potential energy, the Schrödinger equation for strained Ge materials has the following form:

$$
\left\{-\frac{\hbar^{2}}{2 m_{0}} \nabla^{2}+U_{\text {unstrain }}(r)+U_{\text {Deformation }}(r)\right\} \Psi(r)=\varepsilon \Psi(r) .
$$

In formula (1), $U_{\text {unstrain }}(r)$ represents the lattice periodicity potential field of relaxed Ge material, and $U_{\text {Deformation }}(r)$ represents the lattice deformation potential field. The eigen function of (1) has the form of a Bloch wave function, which is $\Psi_{n k}(r)=e^{i k \cdot r} u_{n k}(r)$, where the wave vector $k$ varies throughout the Brillouin region.

Applying the steady-state perturbation theory, the zero-order wave function is used to expand any one of the extreme values of the conduction band energy valley. Finally, the analytical model of the conduction band $E-k$ relationship of the strained Ge material is obtained as follows:

$$
E^{i}(k)=E_{c}\left(k_{0}\right)+U_{k_{0}^{i}}+\frac{\hbar^{2}}{2}\left[\frac{\left(k_{x}-k_{0 x}^{i}\right)^{2}}{m_{t}^{*}}+\frac{\left(k_{x}-k_{0 y}^{i}\right)^{2}}{m_{t}^{*}}+\frac{\left(k_{x}-k_{0 z}^{i}\right)^{2}}{m_{l}^{*}}\right] .
$$

In the formula, $E_{c}\left(k_{0}\right)$ is the bottom energy valley energy level of the relaxed $\mathrm{Ge}$ conduction band, $\left(k_{0 x}^{i}, k_{0 y}^{i}, k_{0 z}^{i}\right)$ is the $k$-vector position of the bottom energy valley energy level of the conduction band, $m_{t}^{*}$ is the longitudinal effective electron mass, and $m_{l}^{*}$ is the transverse electron effective mass. $U_{k_{0}^{i}}$ can be obtained from the deformation potential theory:

$$
H_{\varepsilon, v}=\Xi_{d}\left(\varepsilon_{x x}+\varepsilon_{y y}+\varepsilon_{z z}\right)+\Xi_{d} \varepsilon_{v v}
$$

Among them, $v=x, y, z, \Xi_{d}$ and $\Xi_{u}$ are deformation potential constants, $\varepsilon_{x x}, \varepsilon_{y y}, \varepsilon_{z z}, \varepsilon_{x y}, \varepsilon_{x z}, \varepsilon_{y z}$ are strain tensors, and their expressions are determined by the deformation potential model. The strain tensor results of the (001) substrate, (101) substrate, and (111) substrate are shown in (4) to (6) below, where the parameters in the formula are arranged in Table 1. 


$$
\begin{aligned}
& \left\{\begin{array}{l}
\varepsilon_{x x}=\varepsilon_{y y}=\frac{c_{11}}{c_{11}^{2}+c_{11} c_{12}-2 c_{12}^{2}} T, \\
\varepsilon_{z z}=-\frac{2 c_{12}}{c_{11}^{2}+c_{11} c_{12}-2 c_{12}^{2}} T, \\
\varepsilon_{x y}=0, \varepsilon_{z x}=0, \varepsilon_{y z}=0,
\end{array}\right. \\
& \left\{\begin{array}{l}
\varepsilon_{x x}=\varepsilon_{z z}=\frac{c_{11}-2 c_{12}}{2\left(c_{11}^{2}+c_{11} c_{12}-2 c_{12}^{2}\right)} T, \\
\varepsilon_{y y}=\frac{c_{11}}{c_{11}^{2}+c_{11} c_{12}-2 c_{12}^{2}} T, \\
\varepsilon_{x y}=0, \varepsilon_{z x}=-\frac{1}{2 c_{44}} T, \varepsilon_{y z}=0,
\end{array}\right. \\
& \left\{\begin{array}{l}
\varepsilon_{x x}=\varepsilon_{y y}=\varepsilon_{z z}=\frac{2\left(c_{11}-c_{12}\right)}{3\left(c_{11}^{2}+c_{11} c_{12}-2 c_{12}^{2}\right)} T, \\
\varepsilon_{x y}=\varepsilon_{z x}=\varepsilon_{y z}=-\frac{1}{3 c_{44}} T .
\end{array}\right.
\end{aligned}
$$

Based on the analytical model of the $E-k$ relationship of the strained Ge conduction bands of (1) and (2), the functional relationship between the physical parameters of the strained Ge energy bands and the changes in stress can be drawn. Figure 3 shows the conduction band energy band diagram of the s-Ge/Si device and the corresponding electron effective mass isoenergy (the constant energy contours).

Figure 3 shows the energy levels of the strain energy conduction band $\Gamma$ energy valley, $L$ energy valley, and $\Delta$ energy valley. When the relative strain intensity on the abscissa is about $0.21 \%$, the ordinate corresponds to the energy level values of the strain Ge conduction band energy valley. The two figures on the right correspond to the effective energy isoenergy diagram of the $L$ energy valley electron are ellipsoidal; the effective energy isoenergy diagram of the $\Gamma$ energy valley electron is spherical, and its effective mass is shown to be isotropic.

The simulation method of Monte Carlo is used to establish its electron mobility model. It is a numerical analysis method commonly used in the research of semiconductor carrier transport mechanisms. The effect of some random independent events (such as scattering events) on the carrier transport results is simulated by randomly taking some parameters within a certain range. Since the Schottky diode is a majority carrier device, the electron mobility is considered. For the s-Ge/Si electron scattering mechanism, there are mainly the following: lattice scattering, ionized impurity scattering, and intervalley phonon scattering $[12,13]$ :

$$
\begin{aligned}
& P_{\mathrm{ac}}=\frac{2^{1 / 2} m^{3 / 2} k_{B} T_{L} \Xi^{2}}{\pi \hbar^{4} c_{L}} E^{1 / 2}, \\
& P_{\mathrm{II}}=\frac{\sqrt{2} N_{i} e^{2} m^{3 / 2} \sqrt{E} K_{B}^{2} T^{2}}{\pi \hbar^{2} n\left(8 m \varepsilon_{0} \varepsilon K_{B} T E+\hbar^{2} e^{2} n\right)},
\end{aligned}
$$

TABle 1: Coefficient of elastic stiffness of IV semiconductor material.

\begin{tabular}{lccc}
\hline$d y n / \mathrm{cm}^{2}\left(\times 10^{11}\right)$ & $C_{11}$ & $C_{12}$ & $C_{44}$ \\
\hline $\mathrm{Si}$ & 16.56 & 6.39 & 7.95 \\
$\mathrm{Ge}$ & 12.87 & 4.77 & 6.67 \\
\hline & & & \\
$P_{\text {in }}=\frac{D_{i}^{2}\left(m^{*}\right)^{3 / 2} Z_{f}}{2^{1 / 2} \pi \hbar^{3} \rho \omega_{i}}\left(N_{i}+\frac{1}{2} \mp \frac{1}{2}\right)\left(E \pm \hbar \omega_{i}-\Delta E_{f i}\right)^{1 / 2} \cdot$ &
\end{tabular}

In equations $(7) \sim(9) P_{\mathrm{ac}}, P_{\mathrm{II}}$, and $P_{\mathrm{in}}$, respectively, represent acoustic phonon scattering, ionized impurity scattering, and intervalley phonon scattering; $\Xi$ is the deformation potential constant, and $m^{*}$ is the effective mass of the density of electronic states. Table 2 shows the specific values of other parameters.

This paper uses Monte Carlo method and electron scattering mechanism to simulate the carrier transport process. The result is shown in Figure 4.

Figures 4(a) and 4(b) show the relationship between the average electron energy and drift velocity in s-Ge/Si with the applied electric field strength under different strain levels. As shown in Figures 4(c) and 4(d), at the same energy level, the scattering rate of the ionized impurity in the conduction band of the strained Ge material decreases with the increase of the tensile strain. In contrast to the acoustic phonon scattering, electron acoustic phonon scattering rate of the conduction band of strained Ge material increases with increasing tensile strain. It can be seen from Figure 4(e) that, with the increase of the electric field strength, the average electron energy also increases, and the electron drift speed increases rapidly before the electric field strength reaches the order of $10^{3} \mathrm{~V} / \mathrm{cm}$, and then gradually becomes saturated. However, under different strains, the variation curves slightly increased, and the values on the curves produced random fluctuations. As shown in Figure 4(f), the relationship between the conduction band electron mobility in $\mathrm{s}-\mathrm{Ge} / \mathrm{Si}$ and the strain is obtained. The conduction band mobility of the strained Ge material increases only slightly with the increase of the stress during the tensile loading, and the highest is $4130 \mathrm{~cm}^{2} / \mathrm{Vs}$. Comparing $4(\mathrm{e})$ and $4(\mathrm{f})$, we can see that the trends are consistent, as s-Ge/Si devices increase in stress, and their electron mobility also increases.

\section{Strained Ge Semiconductor on Si Substrate Schottky Diode}

According to the kp perturbation theory and Monte Carlo simulation method in the second section, the s-Ge/Si band structure and electron mobility model are established, and the mobility of the epitaxial strain Ge on the Si substrate is $4130 \mathrm{~cm}^{2} / \mathrm{Vs}$. The maximum reverse breakdown voltage of the Schottky diode is designed to be $15 \mathrm{~V}$, and the zero-bias capacitance of the special diode is $0.7 \mathrm{pF}$.

According to the spice parameter of the above device, the physical parameters of the material of the s-Ge/Si Schottky diode can be calculated as follows: 


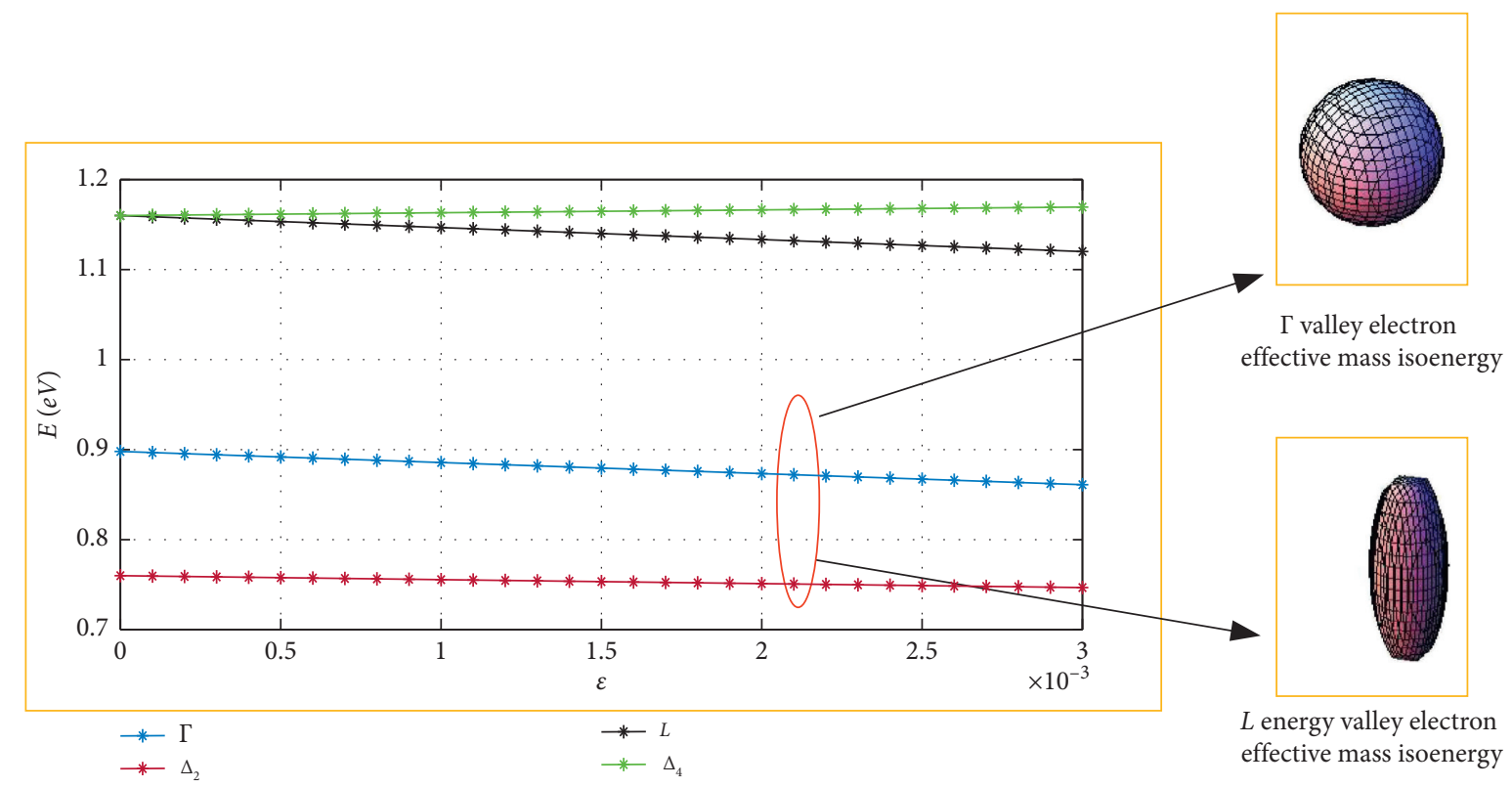

FIgURE 3: Strained Ge conduction band energy valley energy levels and effective electron mass diagrams.

TABLE 2: Parameter table of scattering mechanism.

\begin{tabular}{lcc}
\hline Physical quantity & Symbol & Unit \\
\hline Ionized impurity concentration & $N_{i}$ & $\mathrm{~cm}^{-3}$ \\
Acoustic phonon deformation potential & $\Xi$ & $\mathrm{eV}$ \\
Nonpolar optical deformation potential & $D_{0}$ & $\mathrm{eV} \cdot \mathrm{cm}^{-1}$ \\
Longitudinal elastic constant & $c_{1}$ & $\mathrm{~kg} /\left(\mathrm{m} \cdot \mathrm{s}^{2}\right)$ \\
Dielectric constant of vacuum & $\varepsilon_{0}$ & $\mathrm{~F} \cdot \mathrm{m}^{-1}$ \\
Dielectric constant & $\varepsilon$ & - \\
Long-wave optical phonon energy & $\hbar \omega_{0}$ & $\mathrm{eV}$ \\
Optical phonon number & $n_{o p}$ & - \\
Material density & $\rho$ & $\mathrm{g} \cdot \mathrm{cm}^{-3}$ \\
\hline
\end{tabular}

Doping concentration of the epitaxial layer: $N_{d}=6 \times 10^{-17} \mathrm{~cm}^{-3}$

Minimum epitaxial layer thickness: $t_{\text {epi }} \approx 0.7 \mu \mathrm{m}$

The thickness of the epitaxial layer is calculated to be $0.7 \mu \mathrm{m}$. In order to prevent breakdown of the Schottky diode, the thickness of the epitaxial layer of the Schottky diode is $1 \mu \mathrm{m}$. According to the above spice parameters and physical parameters of the material in s-Ge/Si Schottky diodes, the mobility of Ge is about three times that of Si. In order to ensure a relatively large current, a lateral Schottky diode structure is used. The left side lightly blends with the metal to form a Schottky contact, and the proportion of light blending needs to be properly adjusted. The right side is heavily doped to form an ohmic contact.

The light doping and heavy doping are separated by a silicon dioxide insulating layer, and the distribution of the electric field can be adjusted. To obtain a better currentvoltage curve, the lateral structure of the Schottky diode is used as a buffer layer under light doping and redoping to prevent device breakdown [11-14], and the device structure is simulated in Silvaco TCAD software. The concentration distribution of the device structure is shown in Figure 5.

The s-Ge/Si Schottky diode applies a potential distribution inside the diode when the forward voltage is turned on, as shown in Figure 6.

It can be seen from Figure 6 that, from the anode to the cathode, the potential of inside the device generally decreases first and then increases, and the potential of the heavily doped region remains unchanged. The potential near the anode rises first and then decreases, and this distribution is consistent with the principle of metal to semiconductor contact. At the interface with different doping concentrations, the potential change is obvious because the original Fermi level is different. The substrate layer is charged with a high potential near the anode, and a low potential is applied near the cathode, which tends to become gradually lower.

When a positive voltage is applied according to the s-Ge/ Si Schottky diode anode, the characteristic curve of the device is obtained by scanning the forward voltage as shown in Figure 7.

From the I-V curve of the s-Ge/Si Schottky diode in Figure 7, the internal resistance of the s-Ge/Si Schottky diode can be extracted using the Model Editor of OrCAD software, $R_{\mathrm{s}-\mathrm{Ge} / \mathrm{Si}}=5.2 \Omega$. It can be seen that the internal resistance $R$ of the s-Ge/Si Schottky diode is reduced. Secondly, the reverse breakdown characteristics and $\mathrm{C}-\mathrm{V}$ characteristics of the device can be simulated in Silvaco software, as shown in Figures 8 and 9 .

It can be seen from Figures 8 and 9 that the breakdown voltage of the s-Ge/Si Schottky diode is $B_{V}=15 \mathrm{~V}$, and the zero-bias capacitance is $C_{\mathrm{J} 0}=7 \mathrm{E}-13 \mathrm{~F}$.

In summary, compared with s-Ge/Si Schottky diodes and commercial Ge Schottky diodes, the introduction of 

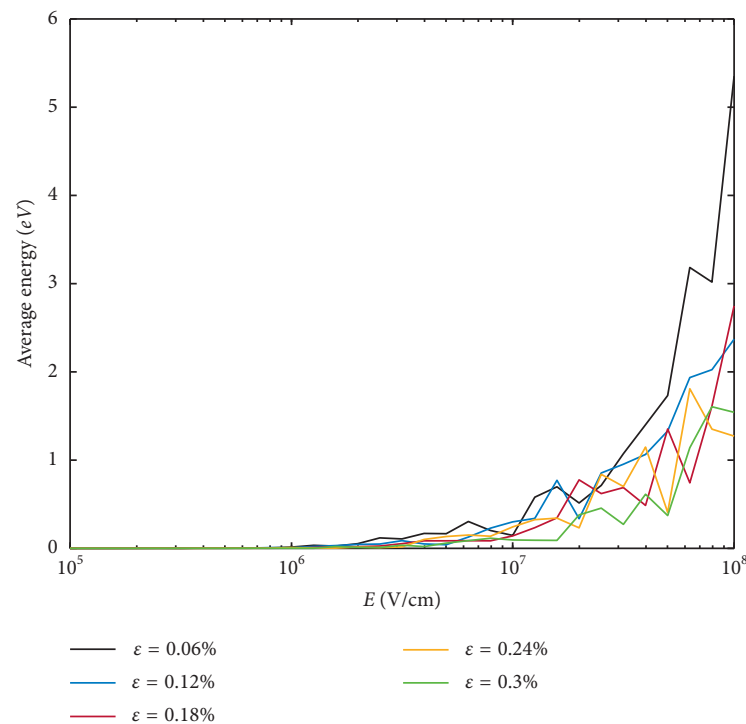

(a)

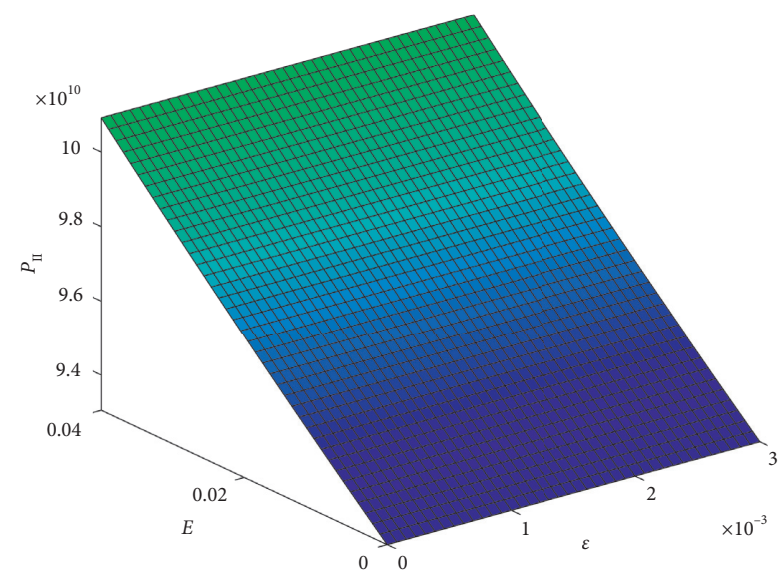

(c)

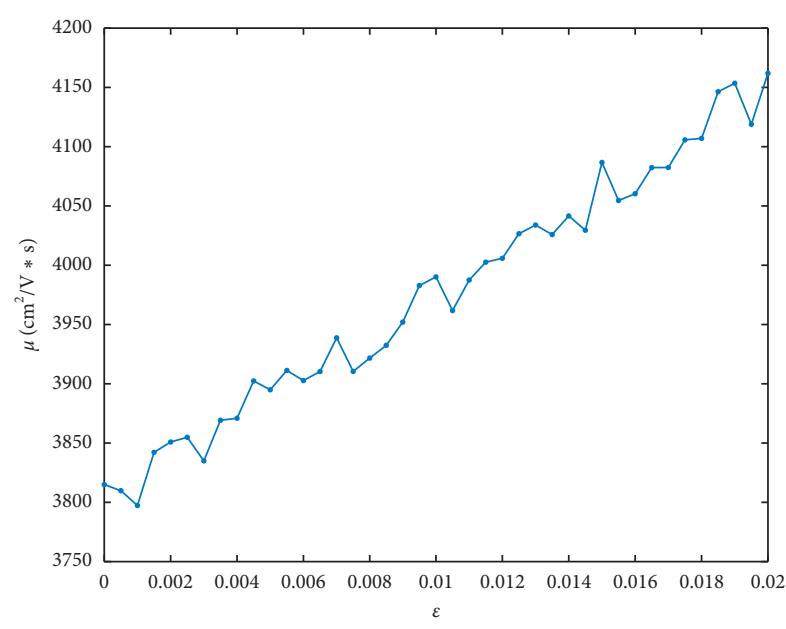

(e)

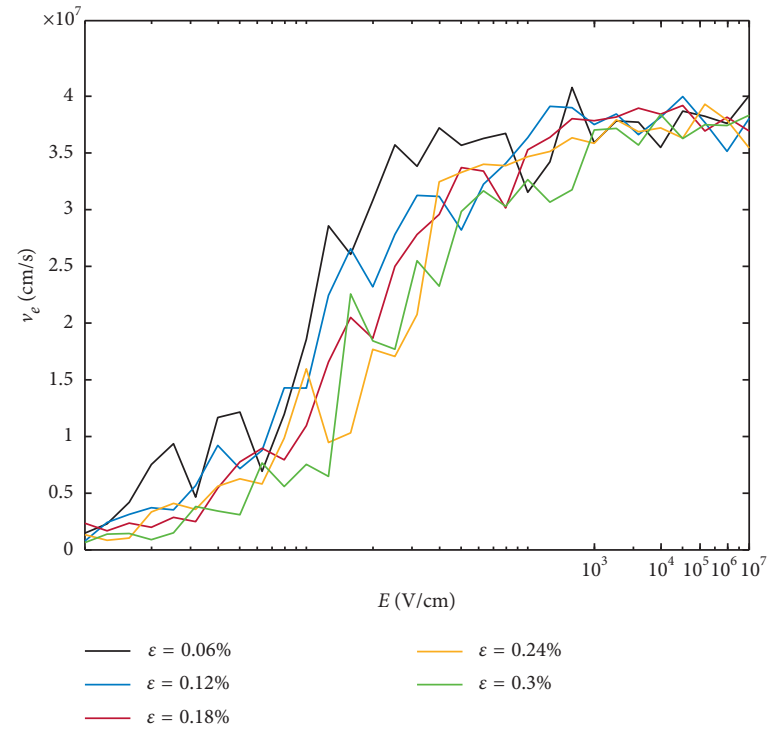

(b)

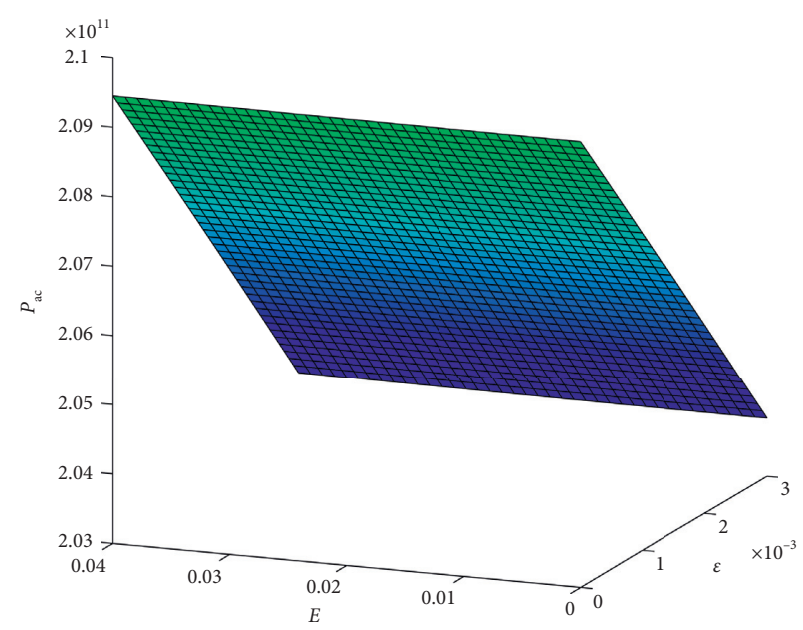

(d)

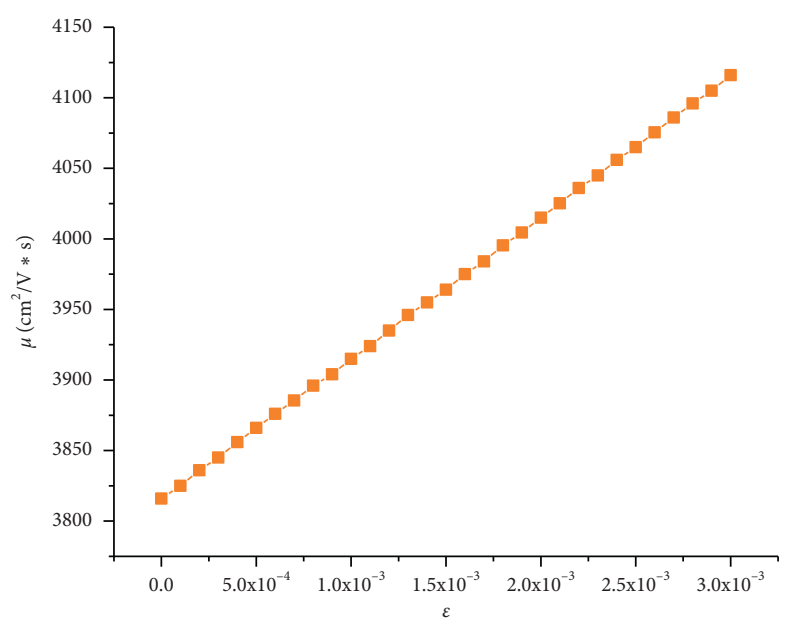

(f)

Figure 4: s-Ge/Si Monte Carlo and electron scattering mechanism simulation carrier transport results. (a) The average electron energy of s$\mathrm{Ge} / \mathrm{Si}$ under different strains varies with the applied electric field. (b) The average electron drift speed of s-Ge/Si under different strains varies with the applied electric field. (c) The relationship between the scattering rate of s-Ge/Si electron ionized impurities and the strain. (d) s-Ge/Si electronic acoustic phonon scattering rate as a function of strain. (e) s-Ge/Si average mobility changes with strain. (f) s-Ge/Si electron mobility as a function of strain. 
ATLAS

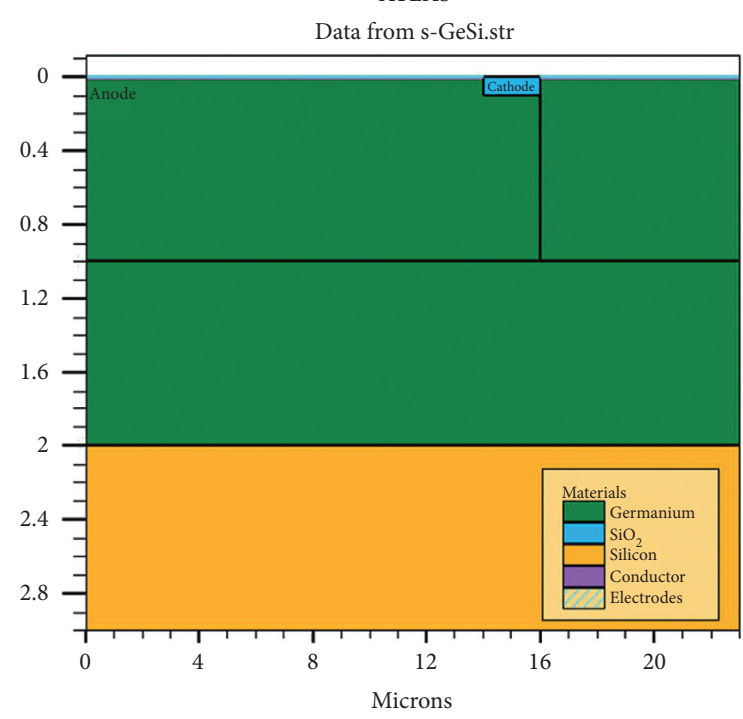

(a)
ATLAS

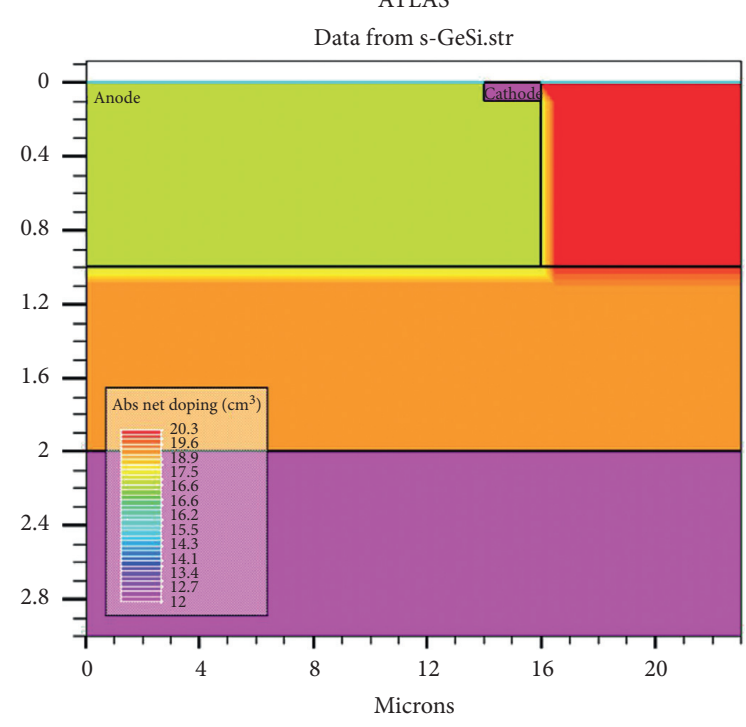

(b)

Figure 5: Structure of epitaxial Ge Schottky diode on Si substrate: (a) s-Ge/Si Schottky diode device structure. (b) s-Ge/Si Schottky diode concentration distribution.

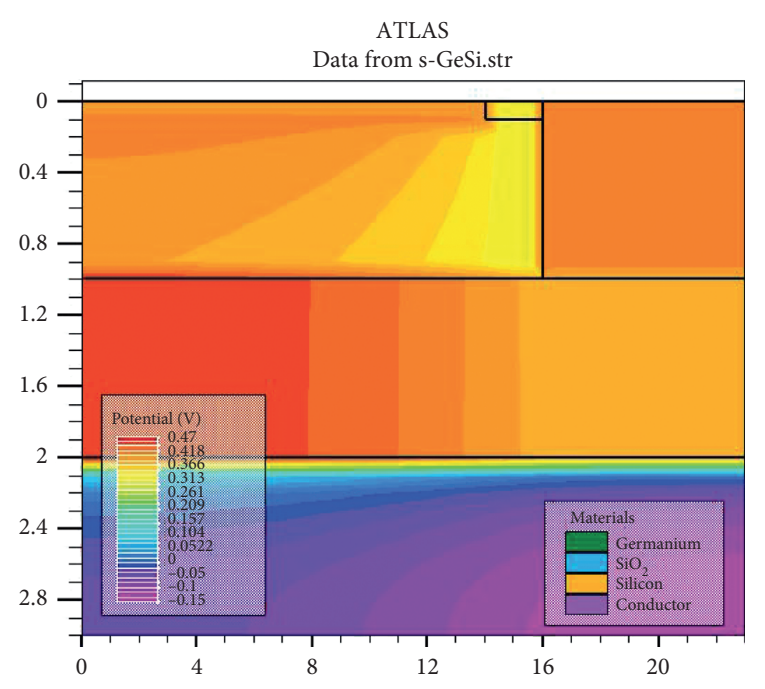

Figure 6: Extension Ge Schottky diode potential distribution on a Si substrate.

strained Ge in the s-Ge/Si Schottky diode device structure proposed in this paper can improve the electron mobility of the device and the internal resistance is reduced. It does not affect the zero offset capacitance and reverse breakdown voltage of the device.

\section{Strained Ge Semiconductor on Si Substrate Schottky Diode ADS Emulation}

According to the s-Ge/Si Schottky diode device structure and device parameters in the third section, the device is used in the rectifier circuit of the MWPT system. Figure 10 shows the design of the rectifier circuit.

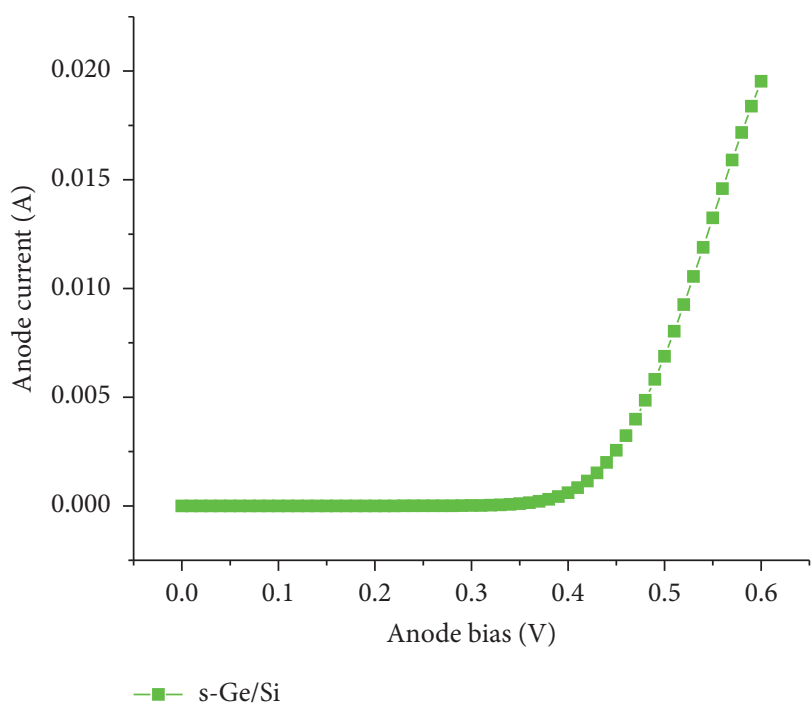

FIgURE 7: s-Ge/Si Schottky diode I-V curve.

$$
\eta_{\mathrm{eff}}=\frac{P_{\mathrm{DC}}}{P_{t}} \times 100 \%
$$

$P_{\text {in }}$ is the maximum possible input power that can be input, $P_{\mathrm{DC}}$ is the power absorbed by the load, $P_{r}$ is the power loss in the case of impedance mismatch, and $P_{t}$ is the input power of the Schottky diode [3]. According to the rectification circuit of Figure 11, ADS software simulation is used to obtain a series load resistor and capacitor with better transmission efficiency in the rectifier circuit. Figure 12 shows the rectification efficiency of different capacitors in the rectifier circuit. Using the impedance self-matching model, the conversion efficiency of the Schottky diode in the rectifier circuit can be defined as follows: 


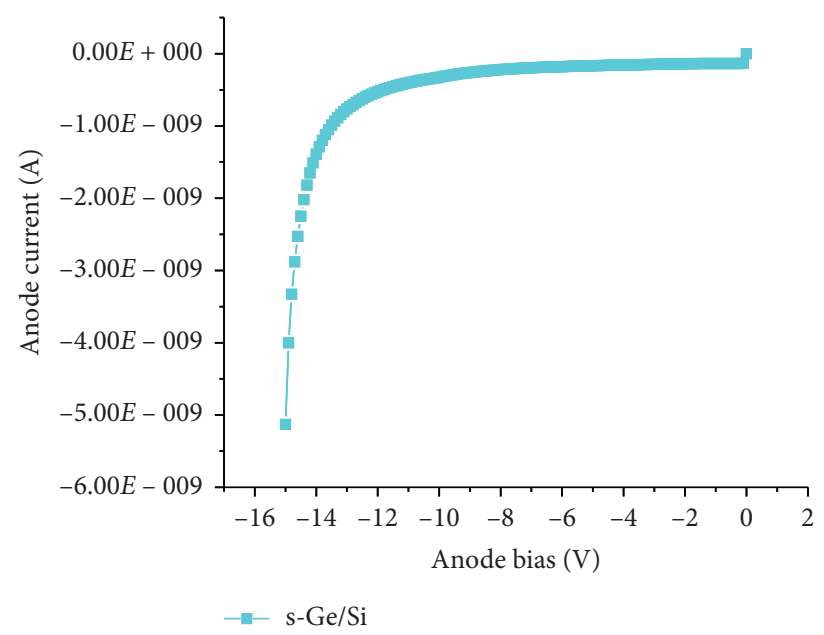

Figure 8: s-Ge/Si Schottky diode breakdown characteristics.

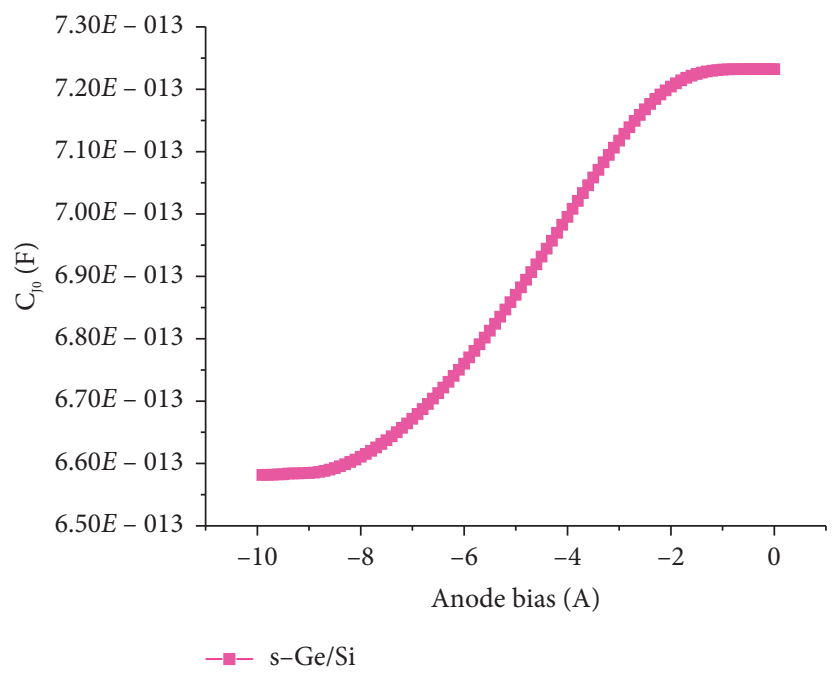

Figure 9: s-Ge/Si Schottky diode C-V curve.

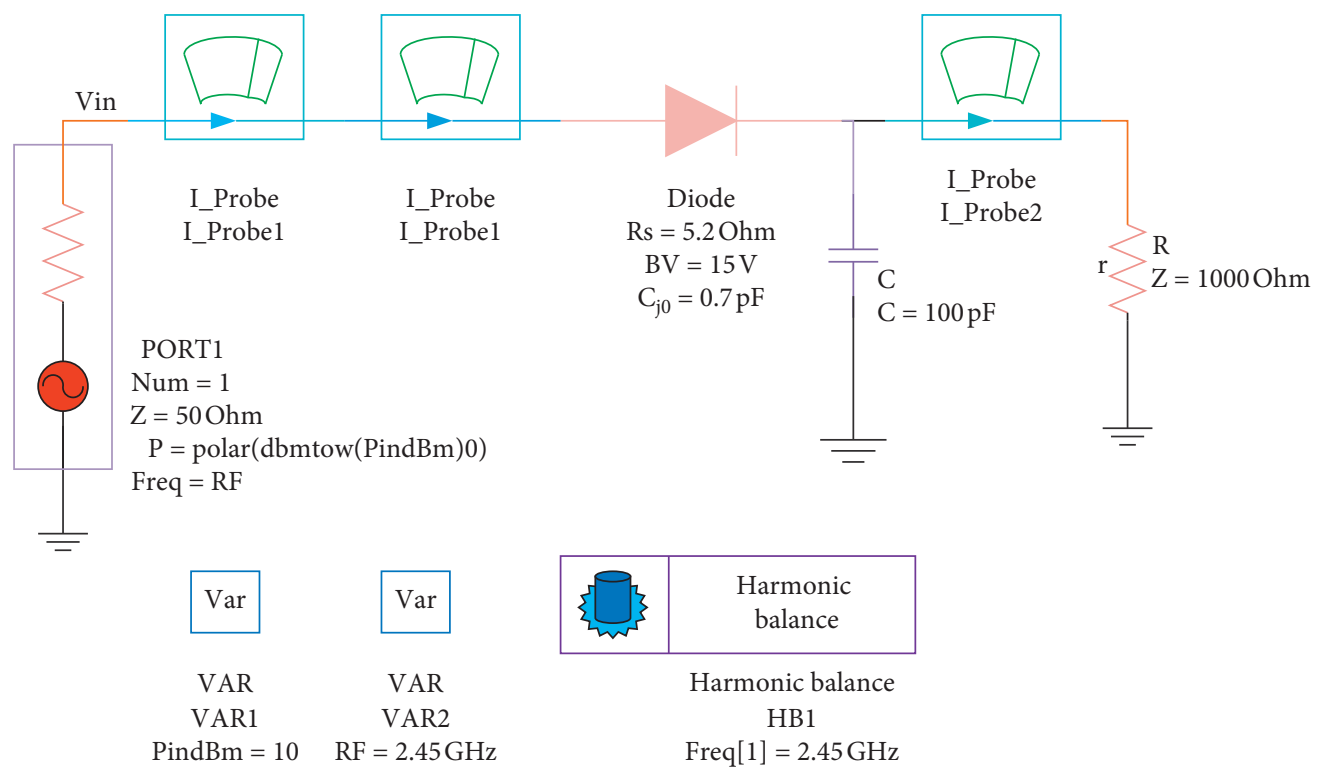

Figure 10: s-Ge/Si Schottky diode harmonic balance. 


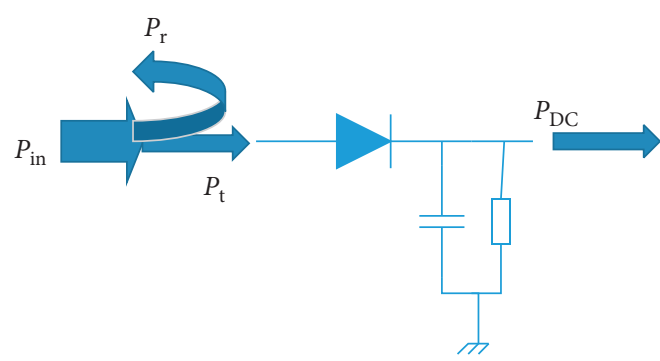

FIGURE 11: Design of the rectifier circuit.

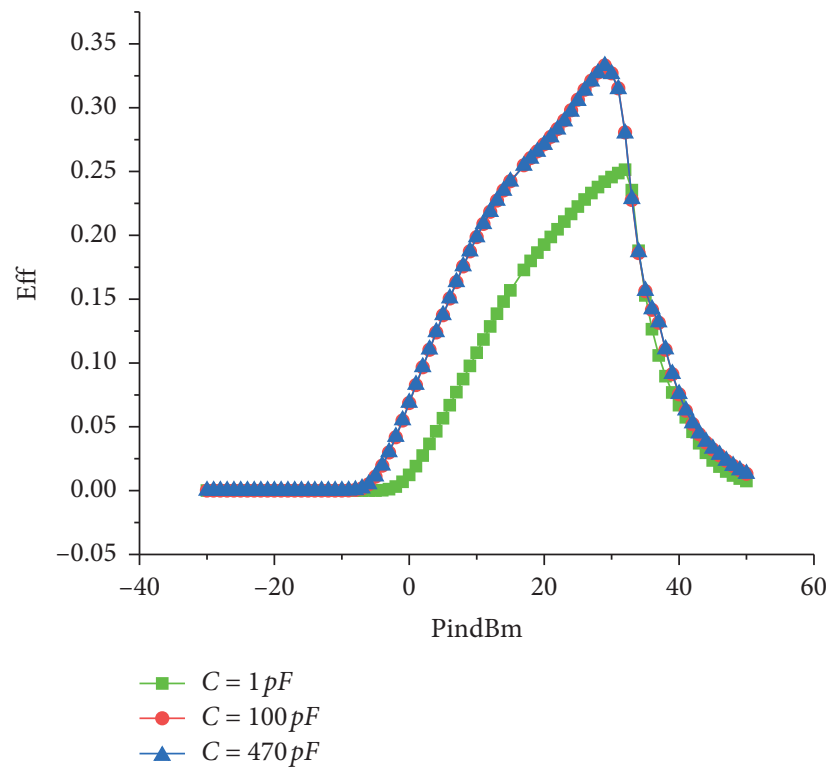

Figure 12: Conversion efficiency of capacitor load $C=1 \mathrm{pF}$, $C=100 \mathrm{pF}$, and $C=470 \mathrm{pF}$ in the rectifier circuit.

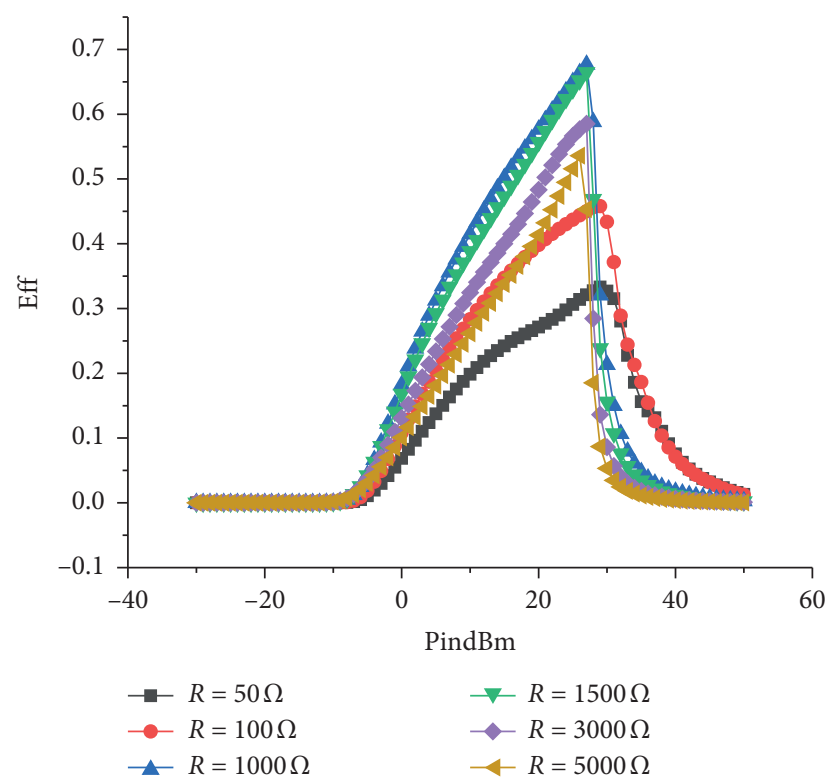

FiguRE 13: Conversion efficiency of resistance load $R=50 \Omega, 100 \Omega$, $1000 \Omega, 1500 \Omega, 3000 \Omega$, and $5000 \Omega$ in the rectifier circuit.

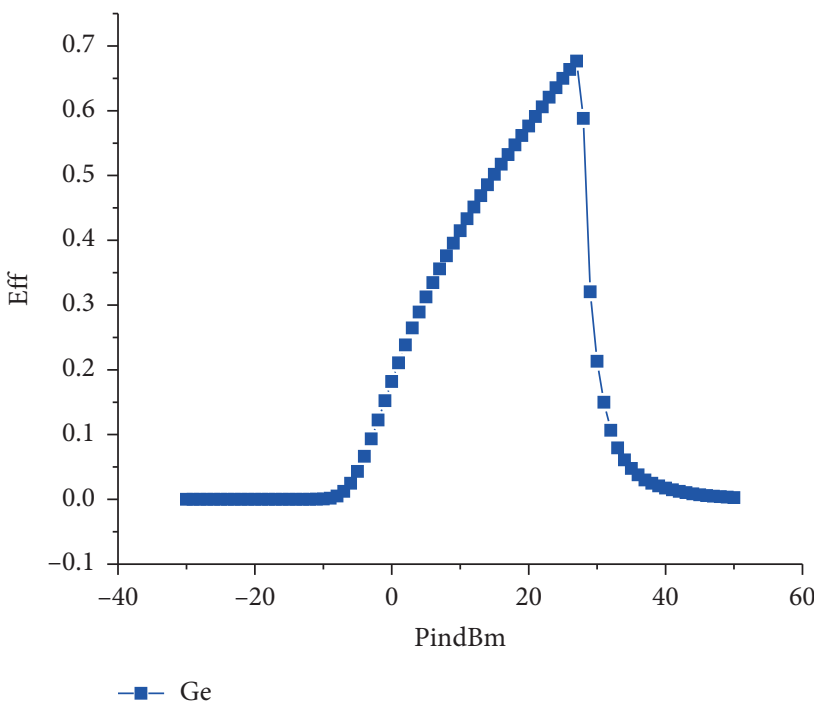

Figure 14: Conversion efficiency of a conventional Ge Schottky diode.

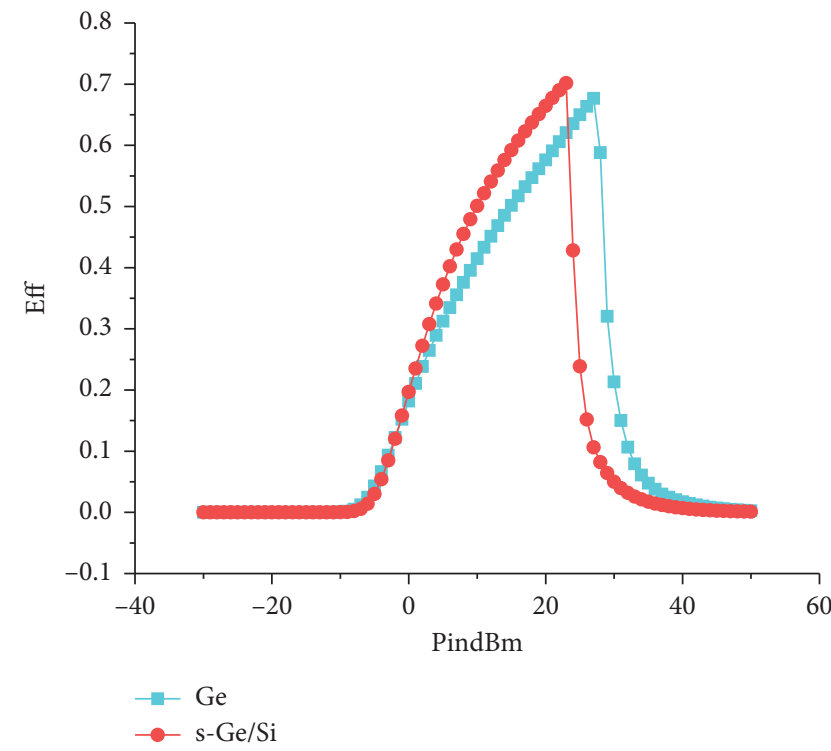

Figure 15: Conversion efficiency of s-Ge/Si Schottky diode in the rectifier circuit.

It can be seen from Figure 12 that when the load resistance is $50 \Omega$, when $C=100 \mathrm{pF}$ and $C=470 \mathrm{pF}$, the conversion efficiency applied in the microwave rectifier circuit is basically the same. When the load capacitance $C=1 \mathrm{pF}$ and $C=100 \mathrm{pF}$, the conversion efficiency in the microwave rectifier circuit is significantly different. The load capacitance $C=100 \mathrm{pF}$ is obviously better than $C=1 \mathrm{pF}$. Therefore, the capacitance load is selected in the microwave rectifier circuit as $C=100 \mathrm{pF}$.

As shown in Figure 13, the better load capacitance is $C=100 \mathrm{pF}$, using different load resistances $R=50 \Omega, 100 \Omega$, $1000 \Omega, 1500 \Omega, 3000 \Omega$, and $5000 \Omega$ in the rectifier circuit, and the conversion efficiency reaches the maximum at $1000 \Omega$. Therefore, the better load in the simulation of the 
ADS microwave rectifier circuit is $R=1000 \Omega$.

In summary, the optimal load capacitance $C=100 \mathrm{pF}$ and the better load resistance $R=1000 \Omega$ are selected in the microwave rectifier circuit of the MWPT system. Rectifier diodes use Agilent's Ge diodes, and the rectification efficiency is obtained as shown in Figure 14.

The s-Ge/Si Schottky diode model is applied to the rectifier circuit, as shown in Figure 10 for the strained Ge Schottky diode harmonic balance simulation. In the ADS software, the $\mathrm{RF}$ frequency is kept at $2.45 \mathrm{GHz}$, the optimal resistance and capacitance are selected to be $1000 \mathrm{Ohm}$ and $100 \mathrm{pF}$ respectively, and then the frequency is linearly harmonic balanced, and the simulation results are shown in Figure 15.

As can be seen from Figure 15, s-Ge/Si is applied to the microwave rectifier circuit, and the conversion efficiency in the rectifier circuit is $70.1 \%$. It can be seen that the epitaxial strain Ge on Si substrate can be applied to rectifier circuits in microwave wireless energy transmission systems, and the conversion efficiency is improved compared to the commercial Ge Schottky diode.

\section{Conclusion}

This paper studies the use of s-Ge/Si Schottky diodes instead of traditional Ge Schottky diodes in rectifier circuits for wireless energy transfer. Firstly, the kp perturbation theory is used to establish the s-Ge/Si band structure model, and the electron mobility model of s-Ge/Si semiconductor is further given. The results show that the introduction of $0.2 \%$ stress in $s-\mathrm{Ge} / \mathrm{Si}$ can increase the electron mobility, and the s-Ge/Si Schottky diodes are based on Si-compatible technology and are easy to integrate. Then, using Silvaco TCAD simulation tool, a relationship model was established between s-Ge/Si Schottky diode performance and device geometry parameters and material physical parameters, and s-Ge/Si Schottky diode device structure is proposed. At the same time, the Schottky diode model is applied to the rectifier circuit of the microwave wireless energy transmission system. The simulation of the rectifier circuit is carried out by using ADS software. Simulation results show that the strained $\mathrm{Ge}$ semiconductor on Si substrate Schottky diode has a rectification efficiency of $70.1 \%$ when the input of the rectifier circuit is $20 \mathrm{dBm}$, the load resistance is $R=1000 \Omega$, and the load capacitance is $C=100 \mathrm{pF}$. Compared with traditional Ge Schottky diodes, this optimal operating point is closer to a low energy density, which is beneficial to a wide range of energy absorption. Finally, the results show that the feasibility of the replacement of Ge Schottky diode can be realized, and the conversion efficiency applied in the rectifier circuit is improved compared with the conventional Ge Schottky diode. Therefore, this paper can provide a valuable reference for the design and development of the core components of MWPT system rectifier circuit.

\section{Data Availability}

All data used to support the findings of this study are available from the corresponding author upon request.

\section{Conflicts of Interest}

The authors declare that there are no conflicts of interest regarding the publication of this paper.

\section{Acknowledgments}

This work was supported by the 111 Project (Grant no. B12026).

\section{References}

[1] H.-H. Ma, "Research on microwave wireless power transmission technology in aerospace," Space Electronic Technology, vol. 3, pp. 57-60, 2013.

[2] G. L. F. Brandão, Ú. C. Resende, F. S. Bicalho, G. A. T. Almeida, and M. M. Afonso, "Parallel association of rectennas for electromagnetic energy harvesting," in Proceedings of the International Symposium on Electromagnetic Fields in Mechatronics, Electrical and Electronic Engineering (ISEF) Book of Abstracts, pp. 1-2, IEEE, Lodz, Poland, September 2017.

[3] F. S. Bicalho, Ú. C. Resende, G. L. F. Brandão, and G. A. T. Almeida, "Analysis of input power dependency for rectennas," in Proceedings of the 3rd Global Electromagnetic Compatibility Conference (GEMCCON), pp. 1-5, IEEE, Sao Paulo, Brazil, November 2017.

[4] K. Huang and V. K. N. Lau, "Enabling wireless power transfer in cellular networks: architecture, modeling and deployment," IEEE Transactions on Wireless Communications, vol. 13, no. 2, pp. 902-912, 2014.

[5] D. Budimir and A. Marincic, "Research activities and future trends of microwave wireless power transmission," in Proceedings of the Sixth International Symposium Nikola Tesla, Belgrade, Serbia, October 2006.

[6] X. Li, L. Yang, and L. Huang, "Novel design of 2.45-GHz rectenna element and array for wireless power transmission," IEEE Access, vol. 7, pp. 28356-28362, 2019.

[7] T. Mori, J. Ida, S. Momose, K. Itoh, K. Ishibashi, and Y. Arai, "Diode characteristics of a super-steep subthreshold slope PNbody tied SOI-FET for energy harvesting applications," IEEE Journal of the Electron Devices Society, vol. 6, pp. 565-570, 2018.

[8] P. M. Nintanavongsa, U. D. R. Lewis, and K. R. Chowdhury, "Design optimization and implementation for RF energy harvesting circuits," IEEE Journal on Emerging and Selected Topics in Circuits and Systems, vol. 1, no. 2, pp. 24-33, 2012.

[9] J. Heikkinen and M. Kivikoski, "Low-profile circularly polarized rectifying antenna for wireless power transmission at $5.8 \mathrm{GHz}$," IEEE Microwave and Wireless Components Letters, vol. 14, no. 4, pp. 162-164, 2004.

[10] W. Yang, J. J. Song, H. Y. Hu, and H. M. Zhang, "Electron conductivity effective mass model for strained-Ge," Journal of Nanoelectronics and Optoelectronics, vol. 13, no. 7, pp. 986994, 2018.

[11] Q. Wei, J. J. Song, C. Q. Zhou et al., "Study of energy band modulation of Ge-based material system for monolithic optoelectronic integration chips," Materials Express, vol. 7, no. 5, pp. 369-379, 2017.

[12] W. Yang, J. J. Song, Y. Ren et al., "Modified Ge energy band structure model for optical devices," Journal of Physics, vol. 67, no. 19, Article ID 198502, 2018.

[13] Z. Liu, X. Hao, A. Ho-Baillie, C.-y. Tsao, and M. A. Green, "Cyclic thermal annealing on $\mathrm{Ge} / \mathrm{Si}(100)$ epitaxial films grown 
by magnetron sputtering," Thin Solid Films, vol. 574, pp. 99-102, 2015.

[14] D. Choi, Y. S. Ge, J. S. Harris, J. Cagnon, and S. Stemmer, "Low surface roughness and threading dislocation density Ge growth on Si (001)," Journal of Crystal Growth, vol. 310, no. 18, pp. 4273-4279, 2008. 